\title{
Multimodal magnetic resonance and fluorescence imaging of the induced pluripotent stem cell transplantation in the brain
}

\section{Yunchao Zhang}

Suzhou Institute of Biomedical Engineering and Technology, Chinese Academy of Sciences

\section{Feifei Wang}

Suzhou Institute of Biomedical Engineering and Technology, Chinese Academy of Sciences

\section{Qing Tao}

Soochow University Affiliated No 1 Peoplel's Hospital: First Affiliated Hospital of Soochow University

\section{Nana Wang}

Suzhou Institute of Biomedical Engineering and Technology, Chinese Academy of Sciences

\section{Xingrui Gi}

Suzhou Institute of Biomedical Engineering and Technology, Chinese Academy of Sciences

\section{Yonggang Li}

Soochow University Affiliated No 1 Peoplel's Hospital: First Affiliated Hospital of Soochow University

\section{Shuang Yu ( $\square$ yush@sibet.ac.cn )}

Suzhou Institute of Biomedical Engineering and Technology https://orcid.org/0000-0002-5604-0293

\section{Jingzhong Zhang}

Suzhou Institute of Biomedical Engineering and Technology, Chinese Academy of Sciences

\section{Research Article}

Keywords: multimodal imaging, magnetic resonance imaging, fluorescent imaging, cell therapy, brain

Posted Date: May 10th, 2021

DOl: https://doi.org/10.21203/rs.3.rs-338518/v1

License: (c) (1) This work is licensed under a Creative Commons Attribution 4.0 International License. Read Full License 


\section{Abstract}

Objectives The understanding of the engrafted cell behaviors is the prerequisite to optimize cell therapy, and a multimodal imaging at both anatomical and molecular levels is designed to achieve this goal.

Results We constructed a lentiviral vector carrying ferritin heavy chain 1 (FTH1), near-infrared fluorescent protein (iRFP) and enhanced green fluorescent protein (EGFP) via T2A linker, and established the induced pluripotent stem cells (iPSCs) culture stably expressing these three reporter genes. These iPSCs showed green and near-infrared fluorescence as well as the iron uptake capacity in vitro. After transplanted the labeled iPSCs into the rat brain, the engrafted cells could be in vivo imaged using magnetic resonance imaging (MRI) and near-infrared fluorescent imaging (NIF) up to 60 days at the anatomical level, moreover, these cells could be detected using EGFP immunostaining and Prussian blue stain at the cellular level.

Conclusions Our study provides a novel tool to study the cellular behaviors of the transplanted cells in a multimodal way, which will be valuable for the effectiveness and safety evaluation of cell therapy.

\section{Introduction}

Cell therapy, promoting the regeneration of dysfunctional or injured tissue by transferring the intact stem cells or their derivatives to the targeted organs, has offered great promise for new medical treatments. Giving the fact that engrafted living cells have the capacity of self-renewal and multidifferentiation, it is of importance to understand the in vivo behaviors of these cells including the survival, growth, distribution and integration.

Researchers have developed various imaging methods to track the engrafted tissue or cells, and each imaging technique has its imaging range, advantages as well as limitations (Zhang et al. 2017; Li et al. 2018). For example, MRI provides excellent soft-tissue contrast and functional, structural and morphological information, however, it could not provide information at the molecular or cellular level (Ngen and Artemov 2017). Optical imaging like green fluorescent protein (GFP) could be imaged at a single cell level, however, the limited penetration depth impedes its application in deep tissue imaging. Therefore, a multimodal imaging method which visualize the molecular and cellular events at different levels is at present a hotspot of the imaging research.

In the recent years, the combination of PET/MRI, BLI/MRI, PET/BLI have been successfully developed and introduced into the clinical application (Li et al. 2018). However, the combination of imaging techniques at both microscale and macroscale levels is still in progress. In the present study, we prepared a lentivirus carrying multimodal reporters FTH1, iRFP and EGFP and established the iPSCs culture stably expressing these labeling genes. We showed that the combination of the three reporters ensured the longterm detection of these transplanted cells by MRI and NIF imaging at the anatomical level, as well as by immunostaining and Prussian blue stain at the cellular level. Our study provides a novel tool to study the 
cellular behaviors of the transplanted cells in a multimodal way, which will be valuable for the effectiveness and safety evaluation of cell therapy.

\section{Materials And Methods}

\section{iPSCs Culture}

iPSCs (kindly provided by Guangzhou Stem cell and Regenerative Laboratory, China) were cultured in Essential 8 (E8) medium (Stem cell, Vancouver, Canada) in 6-well plates with pre-coated Matrigel (Corning, New York, USA). For passaging, the cells were gently washed with phosphate buffer saline (PBS) (Thermo Fisher Scientific, Waltham, MA, USA) $₫$ incubated with cell dissociation solution (PBS containing $1.8 \mathrm{~g} / \mathrm{l} \mathrm{NaCl}$ and $0.5 \mathrm{mM}$ Ethylene Diamine Tetraacrtic Acid (Solarbio, Beijing, China), followed

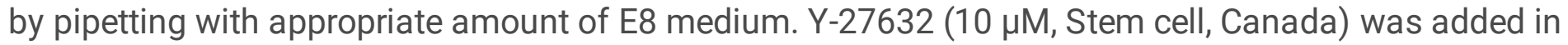
the culture medium after each splitting.

\section{Construction of lentiviral plasmid and package}

The FTH1 fragment was synthesized according to the Genebank sequence NM_012848 (by GENEWIZ Biotechnology company, China) and cloned into the T vector. FTH1 was further amplified to have a fragment with blunt ends by using the primers as follows: 5'-3':

CGGTGAATTCCTCGAGACTAGTTCTAGAGCCACCATGACCACCGCGTCT; 3'-5':

CTCCTCGCCCTTGCTCACCATGATACCGGTAGGGCCGGGATTCTCCTCCA. The fragment was cloned into the plasmid pLVX -EGFP (MiaoLing Plasmid, Wuhan, China) by seamless cloning to construct the plasmid pLVX-FTH1-T2A-EGFP. iRFP was amplified by PCR from the plasmid TET-O-FUW-T2A-iRFP (a kind gift from Prof. Zhang) by using the primers: 5'-3':

CCTGGGACACGGTGATGAGAGCGGATCCGAGGGCAGAGGAAGTCTTCT; 3'-5':

TGTTAGAAGACTTCCTCTGCCCTCAGCGCTCTCTTCCATCACGC. The iRFP fragment was cloned into the plasmid pLVX-FTH1-T2A-EGFP by seamless cloning method to get a final construct pLVX-FTH1-iRFPEGFP.

We used four-plasmids packaging system to package lentivirus. The 293T packing cell line (Cell Bank, Chinese Academy of Sciences) were maintained in the Dulbecco's Modified Eagle medium (DMEM) (Thermo Fisher Scientific, USA) with 10\% (v/v) Fetal Bovine Serum (FBS) (Thermo Fisher Scientific, USA). When the cell density reached $80 \%$, the pLVX-FTH1-iRFP-EGFP and the mixed package plasmids were cotransfected the cells by using Lipofectamine 2000 (Thermo Fisher Scientific, USA). The medium containing virus was collected $48 \mathrm{~h}$ after transfection, followed by centrifugation at $1000 \mathrm{~g}$ for $10 \mathrm{~min}$ at 4 ${ }^{\circ} \mathrm{C}$. The supernatant was collected, and concentrated by using Lenti-x concentration kit (TaKaRa, Japan). The pellets were re-suspended with appropriate amount of PBS (1/10-1/100 of the original volume) and stored at $-80^{\circ} \mathrm{C}$ for further experiment.

\section{Infection of iPSCs with lentivirus}


The lentivirus FTH1-iRFP-EGFP $\left(\mathrm{V}_{\mathrm{fre}}\right)$ was added at a dose of Multiplicity of Infection (MOI) value 2 to 5 together with $2.5 \mathrm{mg} / \mathrm{l}$ Polybrene to the iPSC culture for $12 \mathrm{~h}$. Later, the medium containing virus was removed and fresh E8 medium was added as usual. $V_{\text {fre }}$-infected iPSCs were harvested $48 \mathrm{~h}$ after the infection for the subsequent experiments.

\section{Western blot (WB)}

The sampling of untreated iPSCs, EGFP-infected iPSCs and Vfre-infected iPSCs for WB analysis was performed as described before ( $n=4$ per group) (Chen et al. 2020). Samples were lysed and immunoblotted to detect FTH1 (1:1000; Abcam, Cambridge, UK) andß-Actin (1:1000) (Santa Cruz, Texas, USA). The bands were visualized, scanned and quantified with ImageJ (National Institutes of Health, NIH) after subtraction of local background.

\section{In vitro iron uptake assay}

EGFP-infected iPSCs or $\mathrm{V}_{\text {fre }}$-infected iPSCs were treated with ferric citrate (FAC, Sigma-Aldrich, Shanghai, China) in E8 medium at doses of 0,200 or $500 \mu \mathrm{M}$ respectively. After 5 days of culture, the iPSCs was washed by PBS three times to remove free iron ions. Then $10^{6}$ of iPSCs were collected into a $1.5 \mathrm{ml}$ centrifuge tube and fixed by $10 \mathrm{~g} / \mathrm{l}$ agarose for 15-20 min. The cells were scanned with MRI (MAGNETOM Skyra, SIEMENS, Germany) using the following parameters: Gradient echo, flip angle $20^{\circ}$, repetition time $(T R) /$ echo time $(T E)=3000 / 82 \mathrm{~ms}$, field of view $(F O V)=60 \times 60 \mathrm{~mm}^{2}$, matrix size $=160 \times 120$, slice thickness, $2 \mathrm{~mm}$. The images were analyzed with RadiAnt DICOM Viewer software (Medixant, Poznan, Poland).

\section{$V_{\text {fre }}$-infected iPSCs transplantation into the rat brain}

All animal experiments were performed according to the Regulations in China (Regulations for the administration of affairs concerning experimental animals, 2017) and approved by the Institutional Animal Care and Use committee at Chinese Academy of Sciences. 24 of male SD rats (SPF Biotechnology Co., Ltd. Beijing, China) were subjected to the transplantation surgery and randomly assigned to the subsequent imaging assays. $V_{\text {fre }}$-infected iPSCs were dissociated and re-suspended in saline at a density of $10^{6}$ cells $/ \mu \mathrm{l} .6 \times 10^{6}$ of $V_{\text {fre }}$-infected iPSCs in $6 \mu \mathrm{l}$ of saline were injected into the left striatum of rats at two of the following coordinates (AP: $+1.2 \mathrm{~mm}, \mathrm{ML}:+2.2 \mathrm{~mm}$, DV: -4.0 and $-6.0 \mathrm{~mm}$ ). The same amount of saline was injected into the right striatum at the corresponding coordinates as a self-control for each rat. Transplantation was performed at a rate of $1 \mu \mathrm{l} / \mathrm{min}$ for $3 \mathrm{~min}$, leaving the needle in place for $5 \mathrm{~min}$ and slowly lifting it out. After the surgical procedure, animals were monitored for recovery and returned to the home cage.

\section{In Vivo MRI}

For in vivo $\mathrm{MRI}$, the rats transplanted with $\mathrm{V}_{\text {fre }}$-infected iPSCs were anesthetized with $10 \%$ chloral hydrate, and scanned $3^{\text {rd }}, 9^{\text {th }}, 15^{\text {th }}, 21$ th, $27^{\text {th }}$ and $60^{\text {th }}$ day after the surgery. MRI was conducted at a $3.0 \mathrm{~T} \mathrm{MRI}$ 
scanner using a $2 \mathrm{~cm}$ dual surface coil. T2-weighted signal was acquired using the following parameters to image: Gradient echo, flip angle $20^{\circ}$, TR/TE $=3000 / 82 \mathrm{~ms}, \mathrm{FOV}=60 \times 60 \mathrm{~mm}^{2}$, matrix size $=$ $160 \times 120$, slice thickness, 2 mm. The data were analyzed with RadiAnt DICOM Viewer software.

\section{Near-infrared fluorescence imaging}

The near-infrared fluorescence imaging was performed by using the Kodak In-Vivo MultiSpectral Imaging System FX (Kodak, USA) on the rats 3, 9 and 15 days after the transplantation surgery, respectively. Rats were anesthetized in a holding chamber of the anesthetic gas machine (RWD Life Science, Shenzhen, China) with $40 \mathrm{~g} / \mathrm{l}$ isoflurane (RWD Life Science, Shenzhen, China). The anesthetized animals were placed in the imaging system, and scanned using near-infrared fluorescence at a setting of $663 / 682 \mathrm{~nm}$ (excitation/emission), 60s exposure and $3.0 \mathrm{~cm}$ camera height. A sustained ventilation with 2-3.5\% (v/v) isoflurane was provided during the scanning process to keep the rats still.

\section{Immunohistochemistry and Prussian blue stain}

Rats were perfused with $4 \%$ paraformaldehyde 30 days or 60 days after the transplantation surgery. The brains were carefully removed, dehydrated with sucrose and flash-frozen in dry ice/isopentane and subsequently stored at $-80^{\circ} \mathrm{C}$ until sectioning. Serial coronal cryosection at a thickness of $30 \mu \mathrm{m}$ over the striatum were prepared. Sections were blocked with $30 \mathrm{~g} / /$ Bovine serum albumin (BSA) in PBST, sequentially incubated with antisera against GFP (1:1000, Abcam, Cambridge, UK). Immunoreactivity was visualized using appropriate Alexa Fluor-conjugated secondary antibodies (Invitrogen). DAPI (Beyotime, Shanghai, China) was counterstained for $15 \mathrm{~min}$. Images were acquired with Laser scanning confocal microscope (Zeiss, Jena, Germany).

The cryosections of rat brain were evaluated for iron deposits by using Prussian Blue Staining Kit (Solarbio, Beijing, China). The working solution was prepared by mixing equal volume of $200 \mathrm{~g} / \mathrm{l}$ hydrochloric acid and $100 \mathrm{~g} / \mathrm{l}$ potassium ferrocyanide just before use. Sections were incubated with the working solution, washed and counterstained with nuclear fast red.

\section{Results}

\section{Preparation and identification of the lentivirus carrying multiple reporter genes}

The lentiviral plasmid carrying multiple reporters was constructed by inserting the reporter gene FTH1 and iRFP fragments into the pLVX-EGFP expressing plasmid. To avoid the conformational interaction of the adjacent reporter proteins, a T2A linker was inserted in between two genes (Fig. 1a). To verify if the FTH1

and iRFP fragments were successfully inserted, pLVX-FTH1-iRFP-EGFP was digested by Xbal/BamHI and BamHI/Afel endonucleases, the FTH1 (546bp) and iRFP (948bp) fragments with proper size were observed by electrophoresis (Fig. 1b and c). 
We next tested if the virus carrying multiple reporters could be produced. By introducing the lentiviral plasmid pLVX-FTH1-iRFP-EGFP and helper plasmids into the 293T packaging cell line, we observed clear fluorescence from EGFP and iRFP exogenous proteins in 293T cells (Fig. 1d).

\section{Stable expression of FTH1, iRFP and EGFP reporters in iPSCs}

iPSCs could be obtained from the patient's somatic cells and processed multi-differentiation capacity, thus having great potential in the clinics (Hockemeyer and Jaenisch 2016). To evaluate if lentivirus carrying FTH1, iRFP and EGFP reporter genes $\left(\mathrm{V}_{\text {fre }}\right)$ properly infected iPSCs, $\mathrm{V}_{\text {fre }}$ was introduced to the iPSCs culture and fluorescence of EGFP and iRFP was checked $48 \mathrm{~h}$ after the infection. Both the green fluorescence from EGFP and the red fluorescence from iRFP could be clearly observed in the iPSCs (Fig. 2a), indicating the integration of these two exogenous genes. A further flow cytometric analysis of EGFP fluorescence showed that the EGFP expression was observed in about $34 \%$ of the iPSCs treated with $V_{\text {fre }}$ (Fig. 2b).

The expression of FTH1 in iPSCs was evaluated by WB. iPSCs were infected with either EGFP lentivirus or $V_{\text {fre, }}$ and lysed for WB analysis $48 \mathrm{~h}$ after the infection. As shown in Fig. 2c, there was FTH1 band in the sample from iPSCs infected with $V_{\text {fre }}$, but not in the one from iPSCs/iPSCs infected with EGFP. To evaluate if the iPSCs expressing FTH1 could absorb iron for MRI imaging, we treated the $\mathrm{V}_{\text {fre }}$-infected iPSCs with FAC at different dose. As expected, $\mathrm{V}_{\text {fre }}$-infected iPSCs showed a dose-dependent increase of iron signal in MRI imaging (Fig. 2d), indicating that exogenous FTH1 in iPSCs has the function of iron uptake.

\section{Long-term in vivo imaging of $\mathrm{V}_{\mathrm{fre}}$-infected iPSCs in the brain}

Cell therapy is a promising strategy for many neurodegenerative diseases such as Parkinson's disease (PD) (Ahmadian-Moghadam et al. 2020), meanwhile, the living imaging in the central nervous system remains difficult because of the existence of skull. Therefore, we next tested if the iPSCs carrying multiple reporter genes could be long-term traced in the brain of the living animals. By transplanting the $V_{\text {fre }}{ }^{-}$ infected iPSCs into the striatum of rat brain, we performed MRI and NIF imaging 3, 9, 15, 21, 27 and 60 days after the transplantation. iPSCs were administrated unilaterally, and saline was injected contralaterally as a self-control (Fig. 3a). As shown in Fig. 3b, NIF of iRFP could be observed on the $3^{\text {rd }}$ day after transplantation, however, the iRFP signal diminish rapidly, becoming obscure on the $9^{\text {th }}$ day and invisible on the $15^{\text {th }}$ day (Fig. 3b). In contrast, the iron signal from FTH1 checked by MRI was weak on the $3^{\text {rd }}$ day, and the intensity increased with time; a clear MRI signal from $V_{\text {fre }}$-infected iPSCs could be observed from the $9^{\text {th }}$ day till the $60^{\text {th }}$ day after transplantation (Fig. 3c). Neither near-infrared fluorescence nor iron signal from the control side could be observed (Fig. $\mathbf{3 b}$ and $\mathbf{c}$ ). All of these results indicated that the $V_{\text {fre }}$-infected iPSCs has the potential to be long-term traced in the brain by in vivo imaging.

\section{Histological tracing of the $\mathrm{V}_{\text {fre }}$-infected iPSCs in the brain}


We next checked if $\mathrm{V}_{\text {fre }}$-infected iPSCs could be observed at the cellular level by histological method, after tracing these cells anatomically by MRI and NIF imaging. The rat brain were perfused 30 or 60 days after the transplantation surgery, sectioned and subjected to either EGFP immunostaining or Prussian blue stain. EGFP-immunopositive cells were observed at the transplanting site both 30 and 60 days after the surgery (Fig. 4a), suggesting that these transplanted iPSCs could survive in vivo for at least 2 months. We also found clear iron deposits around the transplanting sites on the $30^{\text {th }}$ and $60^{\text {th }}$ day by using Prussian blue stain (Fig. 4b), which confirmed the validity of our data on MRI imaging. These observations, together with the results of in vivo imaging, indicating that the engrafted iPSCs carrying FTH1, iRFP and EGFP reporters could be long-term traced in the brain, both at the macroscopic and microscopic level.

\section{Discussion}

The ideal imaging method for cell therapy should have the properties of high imaging specificity and sensitivity, with minimal toxicity (Nguyen et al. 2011). Labeling the target cells with lentivirus carrying reporter genes is probably one of the most ideal tools at present (Li et al. 2018). By using a lentiviral vector, the exogenous reporters could be integrated into cell genome and transmitted to the daughter cells, thus providing very specific and stable cell tracking in vivo with little toxicity (Palfi et al. 2018). A combination of multiple reporter genes in one lentiviral vector could further avoid the limitations of a single imaging method and increase the sensitivity at different levels. In the present study, we integrated FTH1, iRFP and EGFP reporters in one lentiviral vector, which made a long-term and specific imaging of the transplanted iPSCs in the brain at both anatomical and molecular levels feasible.

Among the three reporter genes, $\mathrm{FTH} 1$ encodes the heavy subunit of ferritin, which plays a role in the delivery of iron to cells and storage of iron in a soluble and non-toxic state (Chiou et al. 2020). The paramagnetism of iron-accumulating cells reduces the T2-weighted relaxation time, thus showing lower T2-weighted signal than the surrounding tissues in MRI imaging (Dai et al. 2017). EGFP labeling is very specific and non-toxic, having been widely used to for cell tracking (Ansari et al. 2016). However, due to its limited penetration depth, it was hardly detected by in vivo fluorescent imaging (Ansari et al. 2016). iRFP proteins have absorption and emission peaks in the near infrared region, thus having a much better imaging depth and clarity than fluorescent proteins in the visible spectrum (Rogers et al. 2019). A combination of these three genes could therefore realize a simultaneous detection of MRI signal and optical signals from the transplanted iPSCs.

We observed that the signals of MRI and the NIF from the transplanted cells was not completely overlapped. NIF signal was very clear 3 days after the transplantation, becoming faint on the $9^{\text {th }}$ day and invisible on the $15^{\text {th }}$ day. In contrast, MRI signal is not detectable on the $3^{\text {rd }}$ day, starting to be visible on the $9^{\text {th }}$ day and lasting for 60 days. The attenuation of NIF signal indicated that large number of the transplanted iPSCs died shortly after the transplantation surgery, which might because of the injury of surgery and maladaptation to a new niche. Differently, there is a delay of MRI signal since it needs time for FTH1 to accumulate detectable iron from the surrounding tissue. Considering that NIF signal was 
blocked by skull and brain tissue (the imaging depth is $4.0 \mathrm{~cm}$ to $6.0 \mathrm{~cm}$ from the skull), it is reasonable to have the observation that the sensitivity of MRI is superior to that of NIF imaging for tracing cells in the deep brain. These data also suggest that a multimodal imaging model could avoid the limitations of different imaging methods and realize a better trace.

Indeed, various multimodal imaging methods have been developed in the recent years and have exhibited overwhelming superiority to a single imaging method (Li et al. 2018; Lütje et al. 2014; Kim et al. 2010; De Vocht et al. 2011). For example, the targeted radionuclide and fluorescence dual-modality significantly enhance the positioning of the tumor border and effectively guide the surgical resection of the tumor (Lütje et al. 2014). Kim et al developed a bimodal lentiviral vector to monitor deep tissue events using MRI by FTH and BFI by GFP, and showed thatthe MRI and optical imaging can provide an extra level of quantitative and high-resolution information (Kim et al. 2010). In the stem cell transplantation therapy, De Vocht et al. established a combined labeling strategy and proved that labeling of Luciferase/eGFPexpressing bone marrow-derived stromal cells with fluorescent micron-sized iron oxide particles improves quantitative and qualitative multimodal imaging of cellular grafts (De Vocht et al. 2011). Similarly, our study has established a multimodal imaging system which realized simultaneous detection of MRI signal and optical signals including green and NIF, thus providing a new alternative for the cell tracking strategies in the study of cell therapy.

\section{Declarations}

\section{Compliance with Ethical Standards}

\section{Funding}

This study was funded by the Key Research and Development Program of Jiangsu Province, China (Grant No. BE2017669 and BE2018668), the National Nature Science Foundation of China (Grant No. 81701332 to S. Yu), the Key Areas Research and Development Program of Guangdong (2019B020235001), the Major Innovative Research Team of Suzhou, China (Grant No. ZXT2019007) and the Natural Science Foundation of Tianjin (Grant No. 17JCYBJC43400).

\section{Conflict of Interest}

All authors declare no conflict of interest.

\section{Ethical approval}

All animal experiments were performed according to the Regulations in China (Regulations for the administration of affairs concerning experimental animals, 2017) and approved by the Institutional Animal Care and Use committee at Chinese Academy of Sciences.

This article does not contain any studies with human participants. 


\section{References}

1. Ahmadian-Moghadam H, Sadat-Shirazi MS, Zarrindast MR (2020) Therapeutic potential of stem cells for treatment of neurodegenerative diseases. Biotechnol Lett 42:1073-1101.

2. Ansari AM, Ahmed AK, Matsangos AE, et al (2016) Cellular GFP toxicity and immunogenicity: potential confounders in in vivo cell tracking experiments. Stem Cell Rev Rep 12:553-559.

3. Chen Q, Wang F, Zhang Y, et al (2020) Neonatal DEX exposure leads to hyperanxious and depressivelike behaviors as well as a persistent reduction of BDNF expression in developmental stages. Biochem Biophys Res Commun 527.1:311-316.

4. Chiou B, Neely EB, Mcdevitt DS, et al (2020) Transferrin and H-ferritin involvement in brain iron acquisition during postnatal development: impact of sex and genotype. J Neurochem 152:381-396.

5. Dai H, He R, Zhang Y, et al (2017) Adenoviral vector mediated ferritin over-expression in mesenchymal stem cells detected by 7T MRI in vitro. PloS One 12:e0185260.

6. De Vocht N, Bergwerf I, Vanhoutte G, et al (2011) Labeling of luciferase/eGFP-expressing bone marrow-derived stromal cells with fluorescent micron-sized iron oxide particles improves quantitative and qualitative multimodal imaging of cellular grafts in vivo. Mol Imaging Biol 13:1133-1145.

7. Hockemeyer D, Jaenisch R (2016) Induced pluripotent stem cells meet genome editing. Cell Stem Cell 18:573-586.

8. Kim HS, Cho HR, Choi SH, et al (2010) In vivo imaging of tumor transduced with bimodal lentiviral vector encoding human ferritin and green fluorescent protein on a 1.5T clinical magnetic resonance scanner. Cancer Res 70:7315-7324.

9. Li M, Wang Y, Liu M, et al (2018) Multimodality reporter gene imaging: Construction strategies and application. J Theranostics 8:2954-2973.

10. Lütje S, Rijpkema M, Helfrich W, et al (2014) Targeted radionuclide and fluorescence dual-modality imaging of cancer: preclinical advances and clinical translation. Mol Imaging Biol 16:747-755.

11. Ngen EJ, Artemov D (2017) Advances in monitoring cell-based therapies with magnetic resonance imaging: future perspectives. J Int J Mol Sci 18: 198.

12. Nguyen PK, Lan F, Wang $Y$, et al (2011) Imaging: guiding the clinical translation of cardiac stem cell therapy. J Circ Res 109:962-979.

13. Palfi S, Gurruchaga JM, Lepetit $\mathrm{H}$, et al (2018) Long-Term Follow-Up of a Phase I/II Study of ProSavin, a lentiviral vector gene therapy for Parkinson's Disease. Hum Gene Ther Clin Dev 29:148155.

14. Rogers OC, Johnson DM, Firnberg E (2019) mRhubarb: Engineering of monomeric, red-shifted, and brighter variants of iRFP using structure-guided multi-site mutagenesis. Sci Rep $9: 15653$.

15. Zhang W, Zhang S, Xu W, et al (2017) The function and magnetic resonance imaging of immature dendritic cells under ultrasmall superparamagnetic iron oxide (USPIO)-labeling. Biotechnol Lett 39: 1079-1089. 


\section{Tables}

Table 1 Comparison of the direct and reporter-based labeling strategies.

\begin{tabular}{|llll|}
\hline $\begin{array}{l}\text { Imaging } \\
\text { strategy }\end{array}$ & Labeling method & Advantages & Disadvantages \\
$\begin{array}{l}\text { Direct } \\
\text { imaging }\end{array}$ & $\begin{array}{l}\text { Fluorescent dyes, radioactive } \\
\text { substances, magnetic particles } \\
\text { such as SPIO. }\end{array}$ & $\begin{array}{l}\text { 1) Easy to manipulate } \\
\text { 2) Bring no genetic } \\
\text { modification to the } \\
\text { labeled cells. }\end{array}$ & $\begin{array}{l}\text { 1) Signal diluted by cell } \\
\text { proliferation and } \\
\text { phagocytosis. }\end{array}$ \\
& & & $\begin{array}{l}\text { 2) Both viable and dead } \\
\text { cells produce signals. }\end{array}$ \\
& & & $\begin{array}{l}\text { 3) Phagocytosis leads to } \\
\text { unspecific labeling of the } \\
\text { target cells. }\end{array}$ \\
Reporter- & $\begin{array}{l}\text { Introducing exogenous reporter } \\
\text { genes by genetic manipulation. }\end{array}$ & $\begin{array}{l}\text { 1) Specifically label } \\
\text { the target cell types }\end{array}$ & $\begin{array}{l}\text { 1) Introduce genetic } \\
\text { modification to the labeled } \\
\text { cells }\end{array}$ \\
imaging & & $\begin{array}{l}\text { 2) Only viable cells } \\
\text { can be traced in vivo }\end{array}$ & $\begin{array}{l}\text { 2) Rather complex } \\
\text { manipulation }\end{array}$ \\
& & $\begin{array}{l}\text { 3) Signals do not } \\
\text { fade due to cell } \\
\text { proliferation. }\end{array}$ & \\
\hline
\end{tabular}

Table 2 The comparison of the most used in vivo imaging techniques. 


\begin{tabular}{|c|c|c|c|c|}
\hline $\begin{array}{l}\text { Imaging } \\
\text { modality }\end{array}$ & mechanism & Advantages & Disadvantages & Application \\
\hline MRI & $\begin{array}{l}\text { Emitted signal after } \\
\text { nuclear spin } \\
\text { excitation }\end{array}$ & $\begin{array}{l}\text { 1) high penetration } \\
\text { depth }(>500 \mathrm{~mm}) \\
\text { 2) very good soft } \\
\text { tissue contrast } \\
\text { 3) High spatial } \\
\text { resolution }(<100 \mu \mathrm{m})\end{array}$ & $\begin{array}{l}\text { 1) Low sensitivity } \\
(\mu \mathrm{M}-\mathrm{mM}) \\
\text { 2) provide } \\
\text { information at } \\
\text { anatomical level }\end{array}$ & $\begin{array}{l}\text { Imaging at } \\
\text { anatomical } \\
\text { level }\end{array}$ \\
\hline PET/SPECT & Photon emission & $\begin{array}{l}\text { 1) high sensitivity } \\
\text { (nM) } \\
\text { 2) provide quantitative } \\
\text { and tomographic } \\
\text { information } \\
\text { 3) high penetration } \\
\text { depth (>500mm) }\end{array}$ & $\begin{array}{l}\text { 1) poor spatial } \\
\text { resolution (0.5- } \\
2 \mathrm{~mm}) \\
\text { 2) use of } \\
\text { radioactive agents }\end{array}$ & $\begin{array}{l}\text { Imaging at } \\
\text { molecular } \\
\text { level }\end{array}$ \\
\hline $\begin{array}{l}\text { Optical } \\
\text { imaging }\end{array}$ & $\begin{array}{l}\text { Fluorescence or } \\
\text { bioluminescence } \\
\text { light emission }\end{array}$ & $\begin{array}{l}\text { 1) High sensitivity } \\
\text { (nM) } \\
\text { 2) Low cost and high } \\
\text { throughput }\end{array}$ & $\begin{array}{l}\text { 1) Limited tissue } \\
\text { penetration depth } \\
(1-10 \mathrm{~mm}) \\
\text { 2) Low spatial } \\
\text { resolution }(1 \mathrm{~mm})\end{array}$ & $\begin{array}{l}\text { Imaging at } \\
\text { molecular } \\
\text { level }\end{array}$ \\
\hline $\begin{array}{l}\text { Photoacoustic } \\
\text { imaging }\end{array}$ & $\begin{array}{l}\text { acoustic responses } \\
\text { in tissue }\end{array}$ & $\begin{array}{l}\text { 1) high spatial } \\
\text { resolution } \\
\text { 2) low cost }\end{array}$ & $\begin{array}{l}\text { Limited deep } \\
\text { tissue penetration } \\
(5 \mathrm{~cm})\end{array}$ & $\begin{array}{l}\text { Imaging at } \\
\text { anatomical } \\
\text { level }\end{array}$ \\
\hline
\end{tabular}

\section{Figures}



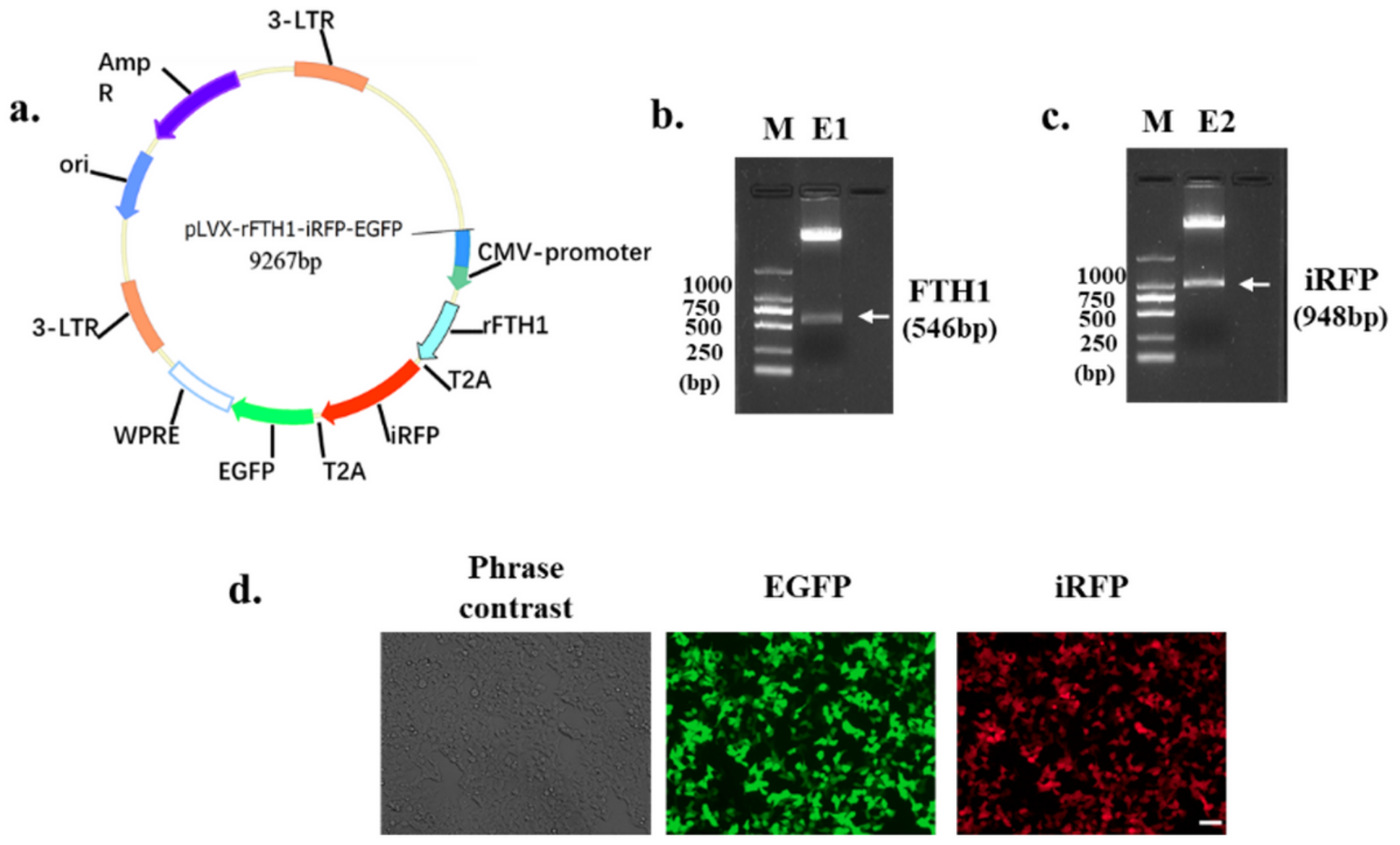

EGFP

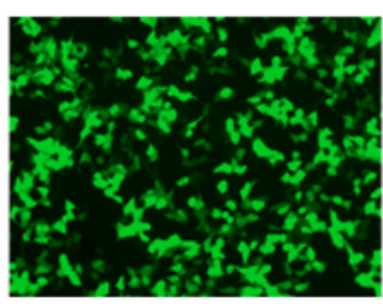

iRFP

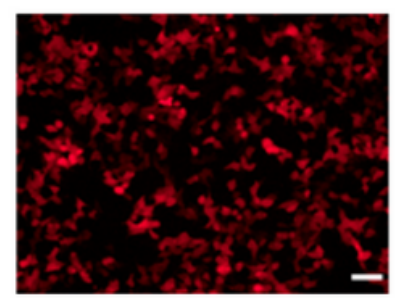

\section{Figure 1}

The preparation of lentivirus carrying FTH1, iRFP and EGFP reporters. a, the map of lentiviral plasmid pLVX-FTH1-iRFP-EGFP. b, the lentiviral plasmid pLVX-FTH1-iRFP-EGFP was digested with Xbal and BamHI, followed by electrophoresis in the lane marked with E1. An expected FTH1 fragment with the size of $546 \mathrm{bp}$ was indicated by white arrows. c, the lentiviral plasmid pLVX-FTH1-iRFP-EGFP was digested with BamHI and Afel, followed by electrophoresis in the lane marked with E2. An expected iRFP fragment with the size of $948 \mathrm{bp}$ was indicated by white arrows. The lane marked with M was loaded with DNA marker 2000. d, the green fluorescence of EGFP and NIF of iRFP could be observed in 293T packing cell line transfected with pLVX-FTH1-iRFP-EGFP lentiviral plasmid. Scale bar: $20 \mu \mathrm{m}$. 
a.

\section{Phrase contrast}
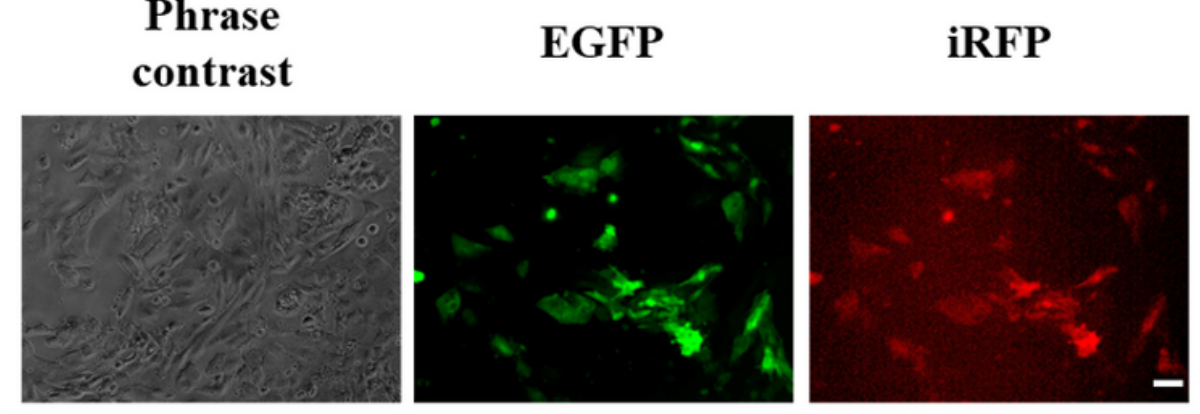

b.

iRFP
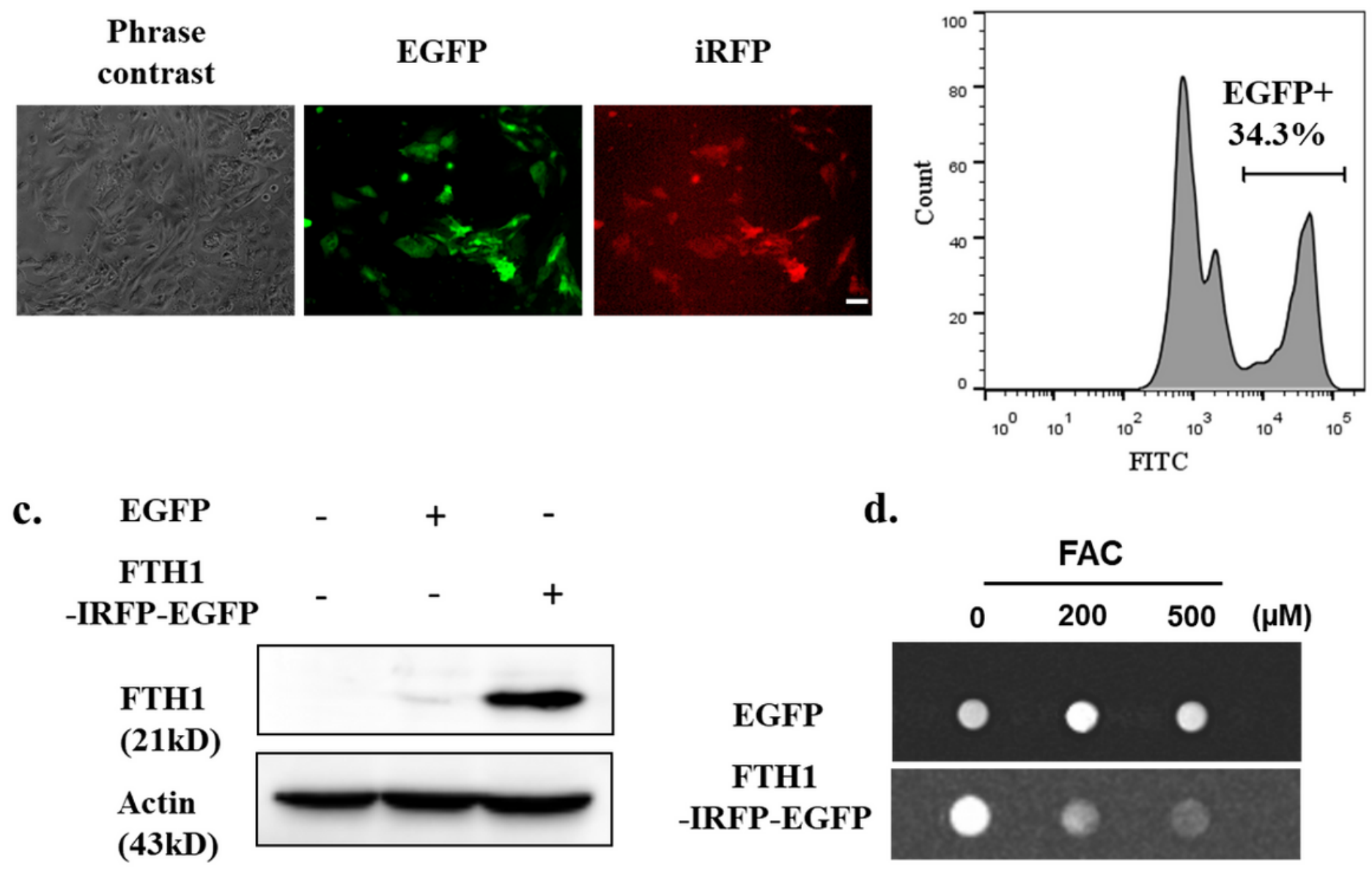

d.

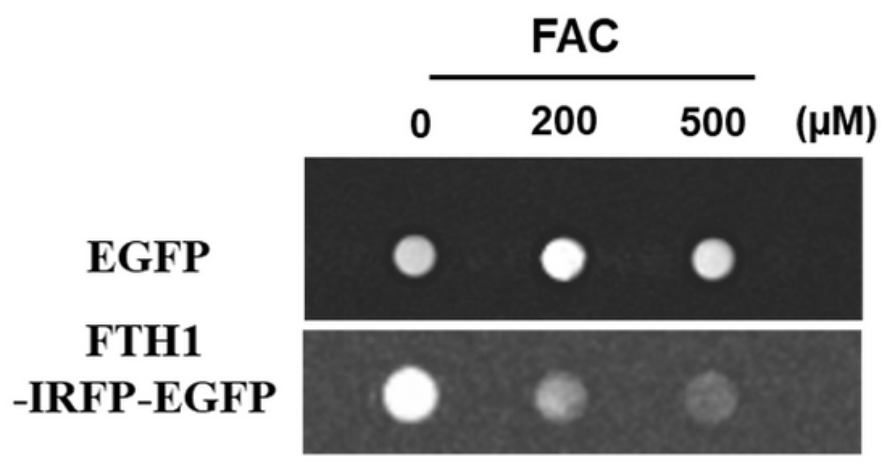

Figure 2

The stable expression of FTH1, iRFP and EGFP reporters in iPSCs. The iPSCs culture was infected with $V$ fre, and examined for fluorescence $48 \mathrm{~h}$ after infection. a, the representative image of Vfre-infected iPSCs under the white light irradiation, as well as under the laser excited at $488 \mathrm{~nm}$ or $650 \mathrm{~nm}$ for EGFP and iRFP detection. $b$, the flow cytometric analysis revealed that the EGFP expression was detected in about $34 \%$ of the iPSCs treated with Vfre. c, WB showing that FTH1 protein was detected only in the iPSCs infected with Vfre. There is FTH1 band neither in the untreated iPSCs or EGFP-infected iPSCs sample subjected for WB. $d$, the representative imaging of MRI showing the iron uptake of Vfre-infected iPSCs treated with FAC at a concentration of 0,200 and $500 \mu \mathrm{M}$. Note a dose-dependent increase of T2weighted signal in Vfre-infected iPSCs with increasing iron concentration, and no signal in the EGFPinfected iPSCs. Scale bar: $20 \mu \mathrm{m}$. 
a.
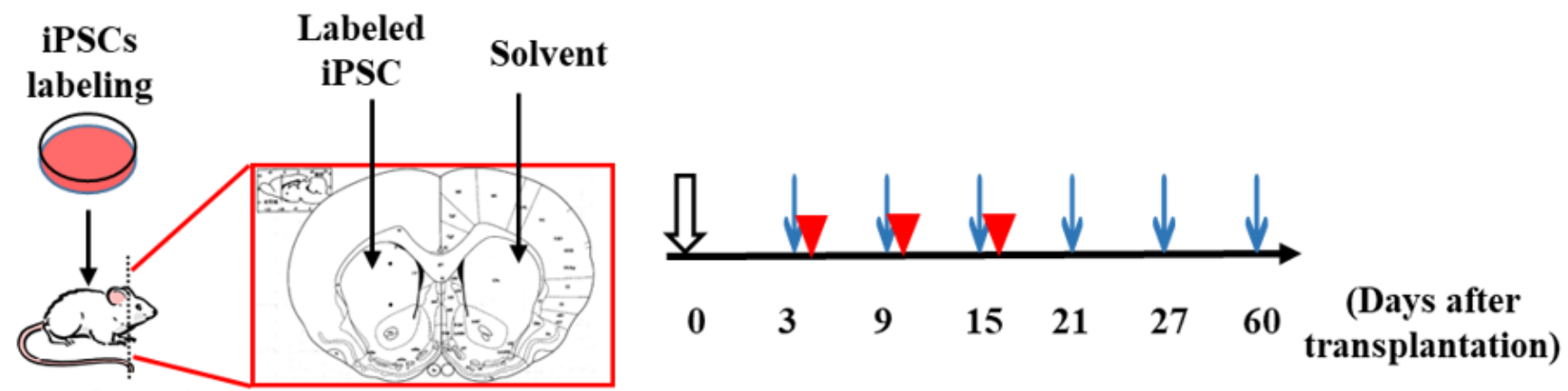

transplantation

b.

D3

D9

D15
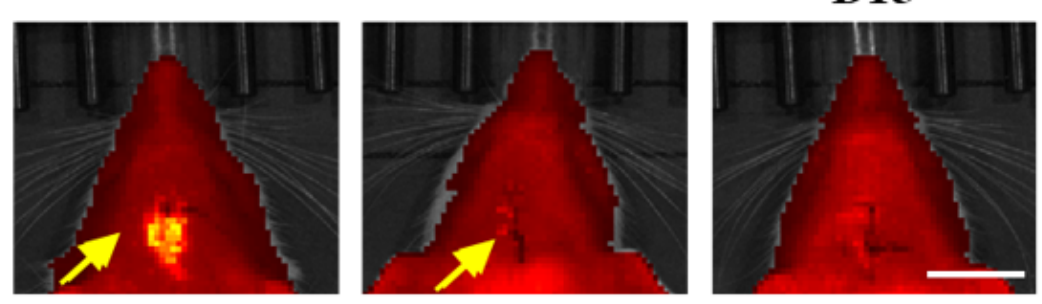

iPSC transplantation

MRI

c.

D3

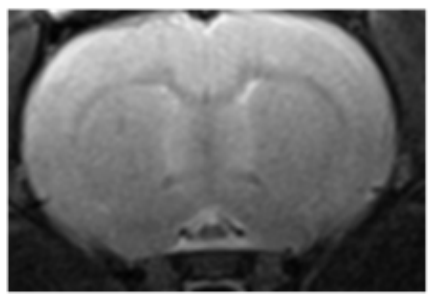

D21

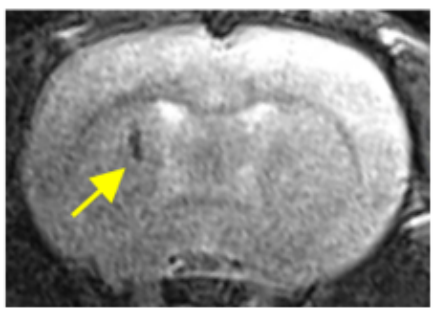

D9

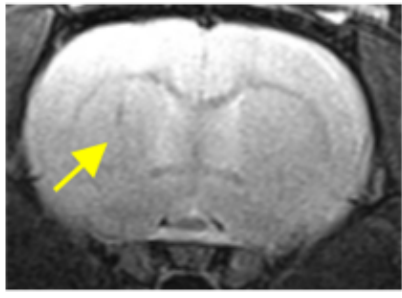

D27

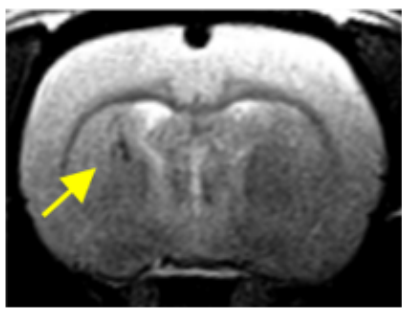

D15

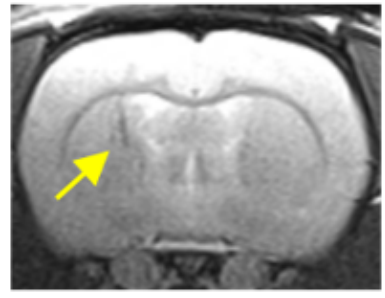

D60

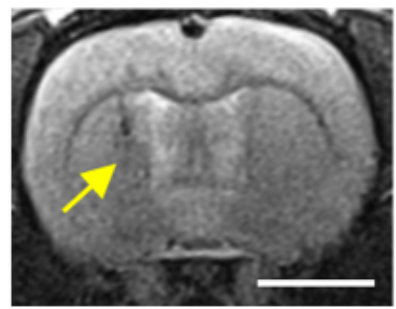

\section{Figure 3}

in vivo imaging of the rat brain transplanted with Vfre-infected iPSCs by MRI and NIF imaging. a, the schematic paradigm of the in vivo imaging experiment. The Vfre-infected iPSCs (about $6 \times 106$ cells) in 6 $\mu \mathrm{l}$ of saline were transplanted into the left side of rat striatum, and another $6 \mu \mathrm{l}$ of saline was injected contralaterally to serve as self-control. b, NIF images showing the in vivo iRFP fluorescence in the brain transplanted with Vfre-infected iPSCs on the 3rd, 9th and 15th day after the surgery. Arrows indicated the iRFP fluorescence from Vfre-infected iPSCs at the transplanting site. c, MRI images showing the T2weighted signal in the brain transplanted with Vfre-infected iPSCs on the 3rd, 9th, 15th, 21th, 27th and 60th day after the surgery. Note that the T2-weighted signal at the transplanting site became visible on the 9th day, and lasted till the 60th day after the transplantation. Arrows indicated the T2-weighted signal 
from Vfre-infected iPSCs at the transplanting site. No such signal could be observed in the right side of the brain injected with saline. Scale bar: $5 \mathrm{~mm}$.

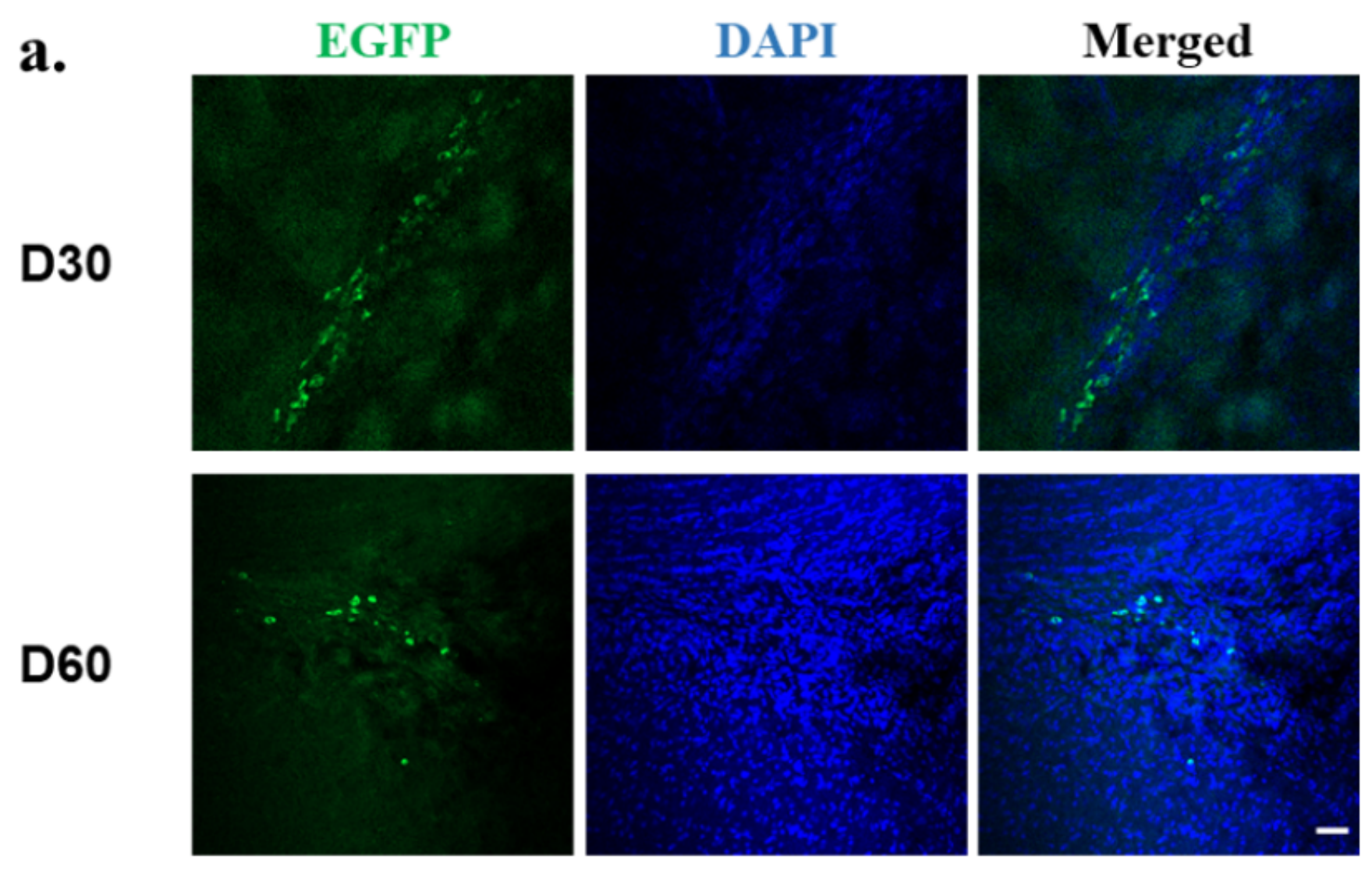

b.

\section{Prussian Blue Staining}

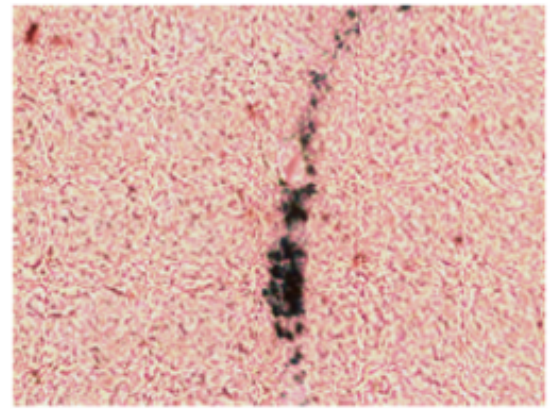

D30

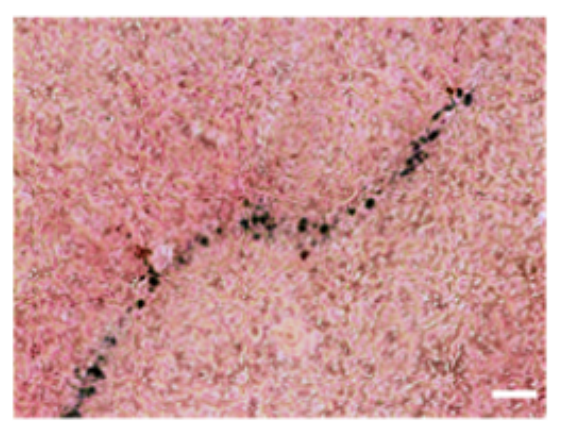

D60

Figure 4

Imaging of the rat brain transplanted with Vfre-infected iPSCs at the cellular level. a, representative microscopic image of the striatum in the rats transplanted with Vfre-infected iPSCs for 30 days (D30) and 60 days (D60); Sections were stained with EGFP antibody and counterstained with DAPI. b, Sections from the striatum of rats transplanted with Vfre-infected iPSCs for 30 days (D30) and 60 days (D60) were 
stained for Prussian blue staining. Iron deposits could be observed both on 30th and 60th day after the transplantation. Scale bar: $50 \mu \mathrm{m}$ in a; $20 \mu \mathrm{m}$ in b. 